\title{
Effect of Temperature on Electrical Conductivity of Zinc Oxide- Polyvinyl Alcohol Nanocomposites
}

\author{
Jayashri Bagawade
}

\begin{abstract}
A set of zinc oxide (ZnO)/polyvinyl alcohol (PVA) nanocomposites were prepared by mixing presynthesized zinc oxide nanoparticles and polyvinyl alcohol (PVA) in different weight percentages with solution casting technique.Different characterization methods are carried out to determine the material properties. The effects of $\mathrm{ZnO}$ nanoparticles loading level on electrical properties of the nanocomposites were investigated in details. The intermolecular interactions within the polymer nanocomposites were explored by FTIR and XRD. The formation of nanocomposites and changes were investigated by SEM analysis.It showed that $\mathrm{ZnO}$ nanoparticles were homogeneously dispersed throughout the whole polymeric matrix. DC conductivity was measured in the temperature range $70-300 \mathrm{~K}$ and it obeys Arrhenius relation. Activation energies were evaluated from Arrhenius plots for all compositions. The samples show a semiconductor-like behavior in regions I (300-130 K) and II (130-70K).The activation energies are smaller at lower temperature range as compared to higher temperature range. The change in conduction mechanism arises at different temperatures, which are feature of the samples. As the ratio of $\mathrm{ZnO}$ to polymer changes these switching positions are shifted to upper temperatures. All the composites obey the Arrhenius equation for the conductivity mechanism which confirms that the nanocomposites are thermally activated. The dc conductivity of all the samples follows a simple hoping type of charge conduction method. This is evident from Fourier Transform Infrared Spectroscopy (FTIR) studies.
\end{abstract}

Keywords: Polymer nanocomposites, ZnO, PVA, dc electrical conductivity

\section{INTRODUCTION}

In the past two decades nanocomposites have become one of the most broadly studied materials due to their remarkable properties and wide range of applications. Polymer nanocomposites have received much interest due to their wide range of potential applications. The development in electrical properties of polymer nanocomposites for diversity of electrical applications have been greatly studied in the past [1-5].The existence of nanofillers in polymer matrix develops and controls the electrical, optical and other properties of the material by its size, morphology and loading concentration. Zinc oxide is a wide band gap material with many promising properties, chemical stability and a high exciton binding energy $(60 \mathrm{meV})$. Recently, $\mathrm{ZnO}$ based polymeric

Revised Manuscript Received on April 25, 2020.

*Correspondence Author Pratishthan's Arts,Science and Commerce College, Vidyanagari, Baramati,

(c) The Authors. Published by Blue Eyes Intelligence Engineering and Sciences Publication (BEIESP). This is an open access article under the CC BY-NC-ND license (http://creativecommons.org/licenses/by-nc-nd/4.0/)
J. A. Bagawade*, Assistant Professor, Department of Physics, Vidya Dist. Pune- 413113 Maharashtra,,India.

nanocomposites are a new class of promising materials to develop new hybrid materials for electrical applications. Polyvinyl alcohol (PVA) is one of the most widely used matrix polymer due to its low cost, aqueous solubility, high dielectric strength, good charge storage capacity, high transparency and dopant reliant electrical properties. The electrical conductivity of polyvinyl alcohol can be altered to a specific requirement by the incorporation of suitable inorganic nanoparticles. Zinc oxide nanoparticles incorporated into the PVA matrix can change or recover the morphological and electrical properties [4]. In the present work, the temperature dependent dc conductivity studies of zinc oxide/polyvinyl alcohol nanocomposites dispersed with different weight percentages of nano-sized $\mathrm{ZnO}$ have been reported.

\section{EXPERIMENTAL}

Basically, capped zinc oxide nanoparticles with a uniform particle size of $~ 3-5 \mathrm{~nm}$ were synthesized, by the simple chemical route [6]. Synthesized zinc oxide nanoparticles were purified and dispersed with polyvinyl alcohol to prepare $\mathrm{ZnO} / \mathrm{PVA}$ nanocomposites. The nanocomposite films of different types were prepared by using casting technique as reported earlier [7]. A known weight of polyvinyl alcohol was dissolved in double distilled water and stirred at $80^{\circ} \mathrm{C}$ for 15 minutes to obtain a viscous transparent solution. The dispersion of zinc oxide nanoparticles was added rapidly to this solution with continuous stirring. The mixture was then heated to make sure complete dissolution and again sonicated for half an hour. Different amounts of $\mathrm{ZnO}$ nanoparticles were used to make the composition of $1: 1$, 2:1, 5:1 ZnO/PVA. The films were then dried at room temperature prior to tests. For reference, neat polyvinyl alcohol film was prepared by spreading PVA/water solution into a dish, followed by solvent evaporation. The polymeric films were prepared. These samples were used for Spectroscopic and electrical conductivity measurements.

\section{RESULTS AND DISCUSSIONS}

The average aggregate size of zinc oxide nanoparticles using TEM were estimated to be $\sim 3$ to $5 \mathrm{~nm}$, which can be found from our previous study [6]. The structural changes of polymer nanocomposites using XRD studies were investigated. It confirmed that the particles in the PVA matrix were zinc oxide nanoparticles. The crystallinity of PVA was substantially influenced by the presence of $\mathrm{ZnO}$ in the nanocomposites.

Published By:

Blue Eyes Intelligence Engineering

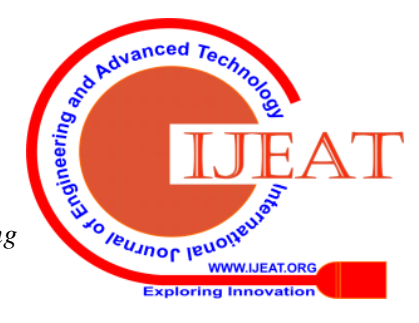


It was detected that the peak positions and their relative intensities altered slightly for various concentrations of nano $\mathrm{ZnO}$ [7]. The SEM micrographs of all these nanocomposite samples were observed by scanning electron microscopy.

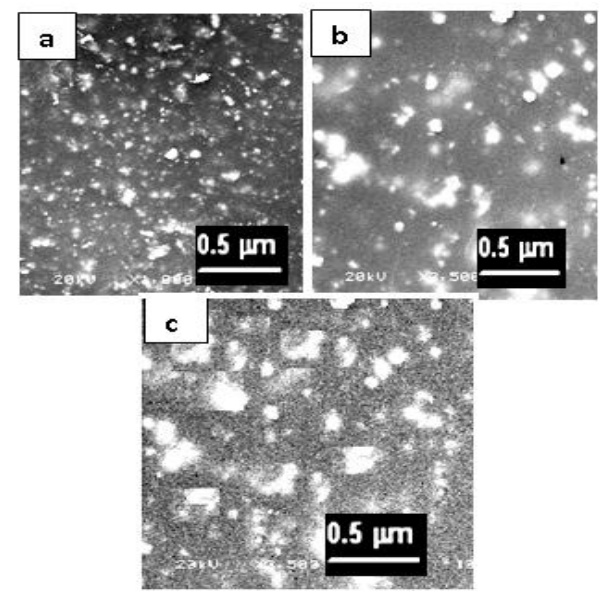

Figure1 SEM images of ZnO/PVA nanocomposites with different concentrations of nano $\mathrm{ZnO}$ (a) 1:1 (b) 2:1(c) 5:1

SEM photographs of the ZnO/PVA nanocomposite films with increased nano zinc oxide concentration are as shown in the Figure1 [7].The films exhibit uniform density of grain distribution in the surface at lower concentrations of $\mathrm{ZnO}$. As, $\mathrm{ZnO}$ concentration increases, the surface morphology of the nanocomposite films shows many aggregates distributed at random. Effectively, the SEM analysis shows an increase of dispersion stability with the increase in $\mathrm{ZnO}$ content in the nanocomposites [7].This confirms higher nano $\mathrm{ZnO}$ concentration lead to the agglomeration of the particles as evidenced in the resistivity data as shown in the resistivity plot. The interaction was further investigated by FTIR. The FTIR spectra of ZnO/PVA nanocomposites with varying nano zinc oxide wt\% showed characteristic peaks of both PVA and ZnO nanofillers [7]. Fourier transform infrared spectrometry analysis as stated in our previous report showed a strong interaction between the zinc oxide nanoparticles and the PVA matrix with a shift of $\mathrm{C}=\mathrm{O}$ (str), stretching vibration of polyvinyl alcohol (PVA) to a higher wave number in these nanocomposites. The analyses exhibited a strong interaction between zinc oxide nanoparticles and PVA matrix [7].The electrical conductivity $(\sigma)$ of the resultant nanocomposites was measured using the standard four-point probe method. The calculated temperature dependent resistivity of the resulting zinc oxide/polyvinyl alcohol nanocomposites was measured between (70-300) K. Resistivity decreases with an increasing the temperature in $\mathrm{ZnO} / \mathrm{PVA}$ nanocomposite samples. The measurements are repeated on every sample and on different samples with same preparation parameters to verify the reproducibility of the measured resistivity data. Although the resistivity values aren't exactly reproducible the same trend of resistivity has been observed for nanocomposites. The data are found to be reproducible within $2 \%$. It can be seen from the study that resistivity of nanocomposites decreased sharply with increasing temperature. The activation energy plots of nanocomposites with different composition are as shown in Figure 2. It shows the variation of the $\ln \sigma(\mathrm{T})$ with $1000 / \mathrm{T}$ for all samples.

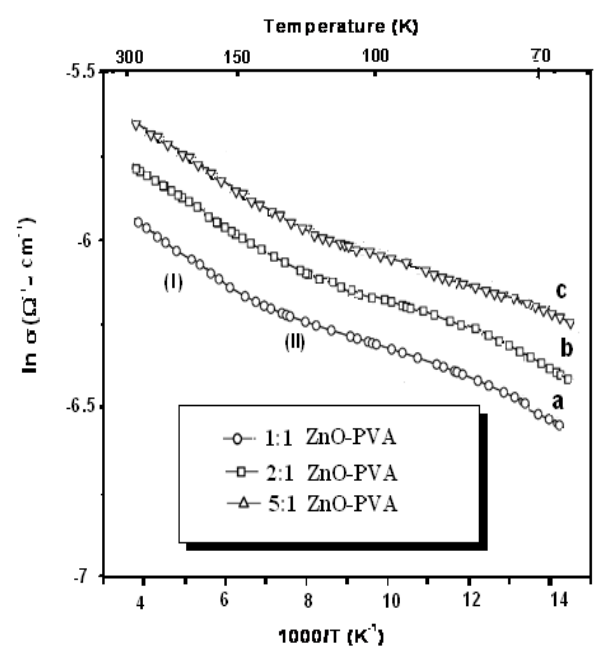

Figure 2 Variation of the $\ln \sigma$ with 1000/T of ZnO/PVA nanocomposites with different concentrations of nano $\mathrm{ZnO}$

(a) $1: 1$ (b) $2: 1$ (c) 5:1

Two different activation regions for two unlike slopes at different temperatures are obtained for all the samples.. i.e. the conduction mechanism changes from one region to another region. Similar to other nanocomposites, the conductivity in these samples is supposed to be an activated one [8-9]. Temperature dependence of the electrical conductivity for semiconductor materials is expressed by the equation: [10]

$$
\sigma=\sigma_{0} \exp \left(\frac{-E a}{K T}\right)
$$

Where $\sigma 0$ is a pre-exponential factor, $\mathrm{T}$ is the absolute temperature in ${ }^{\circ} \mathrm{K}$. and $\mathrm{Ea}$ is the activation energy for electrical process and $\mathrm{K}$ is the Boltzmann constant. By fitting straight lines to the concerned graphs, the activation energies are estimated in different temperature ranges .The activation energy for 5:1 ZnO/PVA sample in 300-130 K (I region) temperature is $\sim 0.592 \mathrm{eV}$, that in the range $130-70 \mathrm{~K}$ (II region) is $\sim 0.050 \mathrm{eV}$ for 2:1 and 1:1 $\mathrm{ZnO} / \mathrm{PVA}$ sample in the temperature range 300-130 $\mathrm{K}$ (I region) are $\sim 0.352 \mathrm{eV}, \sim$ $0.273 \mathrm{eV}$ respectively. While that in the range $130-70 \mathrm{~K}$ (II region) are $\sim 0.045 \mathrm{eV}$ and $0.032 \mathrm{eV}$. All the nanocomposites show a semiconductor-like performance in regions I and II. At lower temperature range the activation energies are smaller in compare to higher temperature range. Thus, the interaction between $\mathrm{ZnO}$ and polymer becomes more prominent at higher temperature. The activation energy increases as $\mathrm{ZnO}$ content increases. i.e. the electrical conduction in these two regions is due to a hopping method between the particles. The particles coalescence which can form bigger islands results into the decrease of activation energy in region II as compared to region I [11]. The activation energy is proportional to the reciprocal of particle size [12]. As the thermal energy is not rather sufficient to activate carriers from these islands the electrical conductivity within this in this temperature range is controlled by carrier production in these huge islands.

Published By:

Blue Eyes Intelligence Engineering

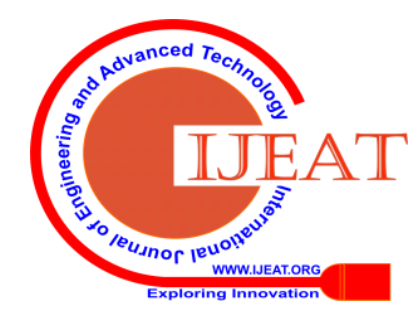


The study of I-V graphs measured at room temperature is carried out for all nanocomposites. The slope of graphs i.e. the resistance of samples, which decreases from $1.9 \times 10^{8} \Omega$ for $1: 1 \mathrm{ZnO} / \mathrm{PVA}$ sample to $0.5 \times 10^{8} \Omega$ for $5: 1$ sample as the ratio of $\mathrm{ZnO}$ to polymer increases. On the contrary at low temperatures the value of resistivity for each sample is decreased by upto two orders $\left(10^{10}\right)$ w.r.t. the value at room temperature. It can be seen from the figure that the samples do not exhibit any metallic resistivity behavior [8].With increasing concentration of $\mathrm{ZnO}$ the resistivity decreases initially at a faster rate then slows down. $\mathrm{ZnO}$ has a very low solubility product constant [13].The conductivity of the nanocomposites mainly comes from the surface charging of the zinc oxide nanoparticles. The fast decrease of resistivity at the initial stage shows more significant aggregation of 2:1or higher wt $\%$ samples compared to $1: 1$ sample. In spite of changing the proportion of zinc oxide to polymer, such an electrical conductivity mechanism exists in other samples. The switching in conduction method occurs at different temperatures, which are feature of the samples. As the ratio of $\mathrm{ZnO}$ to polymer changes these switching points are shifted to higher temperatures. Thus, it may be concluded that observed two slopes in conductivity versus temperature curve may be due to the above two charge transport mechanisms. Such nanocomposites may be used in various fields of electrical or electronic insulation and related areas.

\section{CONCLUSIONS}

Zinc oxide-Polyvinyl alcohol nanocomposites with different weight percentage of $\mathrm{ZnO}$ nanoparticles were prepared by using presynthesized zinc oxide nanoparticles with solution-casting technique. The formation of composites was confirmed by XRD, FTIR and SEM techniques. A standard four-probe method is used to characterize the electrical conductivity of the resulting nanocomposites. Scanning Electron Microscopy (SEM) studies demonstrated the random distribution of zinc oxide nanoparticles for lower concentration. It also showed the formation of nanoclusters for higher concentration of zinc oxide nanoparticles incorporation. The conductivity study revealed that it is thermally activated and show Arrhenius behavior. DC conductivity was found to vary in the temperature range 70-300 K. Activation energy is measured by Arrhenius plots. The samples show a semiconductor-like behavior in regions, I(300-130 K) and II(130-70K ). The interface between ZnO and polymer becomes more prominent at higher temperature. These switching points are transferred to higher temperatures as the ratio of $\mathrm{ZnO}$ to polymer changes. It is also observed that the activation energy increases with the increase in $\mathrm{ZnO}$ content. The switching in conduction mechanism occurs at different temperatures, which are characteristic of the samples. Such polymer nanocomposite materials may be used to develop new hybrid materials for electrical,optical applications and its related areas.

\section{ACKNOWLEDGEMENTS}

I sincerely acknowledge Department of Physics, SPPU for providing required facilities for carrying out this research work.

\section{REFERENCES}

1. S. Ningaraju, H.B. Ravikumar, "Studies on electrical conductivity of PVA/graphite oxide nanocomposites: a free volume approach", J. Polym. Res. 24 (11) 2017, pp.1-11.

2. M.H. Makled, E. Sheha, T.S. Shanap, M.K. El-Mansy, "Electrical conduction and dielectric relaxation in p-type PVA/CuI polymer composite", J. Adv. Res., 4 ,2013,pp.531-538.

3 Jiahua Zhu, Suying Wei, Lei Zhang, Yuanbing Mao, Jongeun Ryu, Amar B. Karki David P. Young and Zhanhu Guo, "Polyaniline-tungsten oxide metacomposites with tunable electronic properties", J. Mater. Chem., 21,2011,pp. 342-348.

4. Agnieszka łodziejczak-Radzimska and Teofil Jesionowski, "Zinc Oxide-From Synthesis to Application: A Review",Materials 7(4) ,2014, pp. 2833-2881.

5. S. Mitra, O. Mondal, D. Ranjan Saha, A. Datta, S. Banerjee, D. Chakravorty, "Magneto dielectric effect in graphene-PVA nanocomposites", J. Phys. Chem. ,115 (2011),pp. 14285-14289.

6. Shriwas Ashtaputre, Aparna Deshpande, Sonali Marathe, M. E. Wankhede, , J. Chimanpure, Renu Pasricha, J. Urban, S. K. Haram, S. W. Gosavi andS. K. Kulkarni.,'Integrating Nano and Microparticles", Pramana Journal of Physics, 165, 2005,pp. 4615

7. Bagawade J.A., "Structural and Spectroscopic Properties of Zno/PVA Nanocomposites",International Journal of Recent Technology and Engineering 8, 3, Sept.2019.pp.2292-2294.

8.. S. Banerjee, D. Chakravorty, "Electrical resistivity of silver-silica nanocomposites”, J. Appl. Phys. 85, 1999,pp. 3623.

9. A. Chatterjee and D. Chakravorty, "Electrical conduction in sol-gel derived glass-metal nanocomposites", J. Phys. D: Appl. Phys. 23, 1990. Pp.1097-1102.

10 S Roy, D Chakravorty, "Electrical properties of sol-gel-derived glass-metal nanocomposites",Journal of Physics: Condensed Matter,6, 1994, ,pp.8599.

11 PA Tick, FP Fehlner, "Electrical behavior of composite discontinuous films", Journal of Applied Physics,43, 1972,pp.362.

12 Herman D.S. and Rhodin T.N., "Electrical Conduction between Metallic Microparticles", J.Appl. Phys., 37 ,1966,pp. 1594

13 Lide, David R, Handbook of Chemistry and Physics, CRC,1998-99

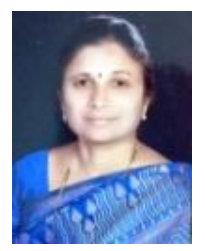

\section{AUTHOR PROFILE}

Dr. Mrs. Jayashri Bagawade, Assistant Professor, at Vidya Pratishthan's Arts, Science and Commerce College, Baramati, India. She received her Ph.D. in Physics, from SPPU,Pune. She published research papers in reputed national and international journals and conferences. Her current research interests are in the field of Material Science, Nanoscience and Nanotechnology, Biophysics, Energy studies etc. 